\title{
Excess pore pressure in a poroelastic seabed saturated with a compressible fluid
}

\author{
Z.Q. YuE \\ Pavements Laboratory, Centre for Surface Transportation Technology, National Research Council of Canada, \\ Ottawa, ON KlA OR6, Canada \\ A.P.S. SELVADURAI \\ Department of Civil Engineering and Applied Mechanics, McGill University, Montréal, PQ H3A 2K6, Canada \\ AND \\ K.T. LAW \\ Department of Civil and Environmental Engineering, Carleton University, Ottawa, ON K1S 5B6, Canada \\ Received August 6, 1993 \\ Accepted July 12, 1994
}

\begin{abstract}
This paper presents an analytical investigation on the excess pore-fluid pressure in a finite seabed layer by taking into account the influence of a compressible pore fluid. The seabed layer is modeled as a poroelastic layer saturated with a compressible pore fluid and resting on a rough, rigid impermeable base. The surface of the poroelastic seabed layer is either completely pervious or completely impervious, and subjected to a normal surface traction induced by offshore structures. The paper presents analytical and numerical results to illustrate the time-dependent behaviour of excess pore pressure in the poroelastic seabed. The results demonstrate that the presence of $a$ compressible pore fluid reduces the generation of excess pore pressure in the poroelastic seabed layer.
\end{abstract}

Key words: excess pore pressure, poroelastic seabed layer, soil consolidation, compressible pore fluid, integral transforms.

Cet article présente une étude analytique de l'excédent de pression interstitielle dans une couche finie de fond marin en prenant en compte l'influence d'un fluide interstitiel compressible. La couche de fond marin est modélisée comme étant une couche poroélastique saturée par un fluide interstitiel compressible ét reposant sur une base imperméable rigide et rugueuse. La surface de la couche de fond marin poroélastique est soit complètement perméable ou complètement imperméable, et est soumise à la traction de surface normalement induite par les structures offshore. L'article présente les résultats analytiques et numériques illustrant le comportement en fonction du temps de l'excédent de pression interstitielle dans la couche poroélastique de fond marin. Les résultats montrent que la présence d'un fluide interstitiel compressible réduit la génération d'excédent de pression interstitielle dans la couche poroélastique de fond marin.

Mots clés : excédent de pression interstitielle, couche poroélastique de fond marin, consolidation des sols, fluide interstitiel compressible, transformées intégrales.

Can. Geotech. J. 31, 989-1003 (1994)

\section{Introduction}

The modeling and prediction of excess pore pressure in seabed soils based on their mechanical behaviour are essential to offshore geotechnical engineering (Poulos 1988). The mechanical behaviour of seabed soils is generally a function of the group action of the particles and the interaction among the solid, fluid, and gas. Under the hypothesis that the porefluid phase and pore-gas phase are mixed in one so-called "gasified" fluid (or air-water mixture), partially saturated seabed soils can be regarded as a quasi-two-phase medium: a solid skeleton containing a compressible fluid (Zaretskii 1972). When loadings are applied to saturated seabed soils (such as clay deposits) by foundations, ground anchors, etc., there is an increase in the pore-fluid pressure. Initially the soil skeleton and structure will undergo an immediate deformation and excess pore pressures will develop. With time, the excess pore pressures will dissipate as pore fluid flows from the regions of high excess pore pressures to the regions of low excess pore pressures. As the excess pore pressures change, Printed in Canada / Imprimé au Canada the stresses and strains in the soil skeleton and structure will simultaneously compensate to maintain the system in equilibrium and to satisfy the conditions of compatibility. This leads to further deformation of soil skeleton and structure. The deformation of soil skeleton will therefore have an effect on the flow of pore fluid and the excess pore pressures, and vice versa. Consequently, the consolidation process of saturated seabed soils is dominated by the time-dependent coupling between the deformation of the solid skeleton and the flow of pore fluid through the voids.

There are several theories that have been developed to idealize mathematically the consolidation behaviour of saturated soils (Terzaghi 1923; Rendulic 1936; Biot 1941, 1956; Zaretskii 1972; Desai and Saxena 1977; Chang and Duncan 1983). Among these theories, the classical linear theory of porous, elastic medium saturated with a compressible fluid developed by Biot $(1941,1956)$ is commonly utilized in the analysis of the soil consolidation phenomenon. This idealized theory is not an exact description of even the 


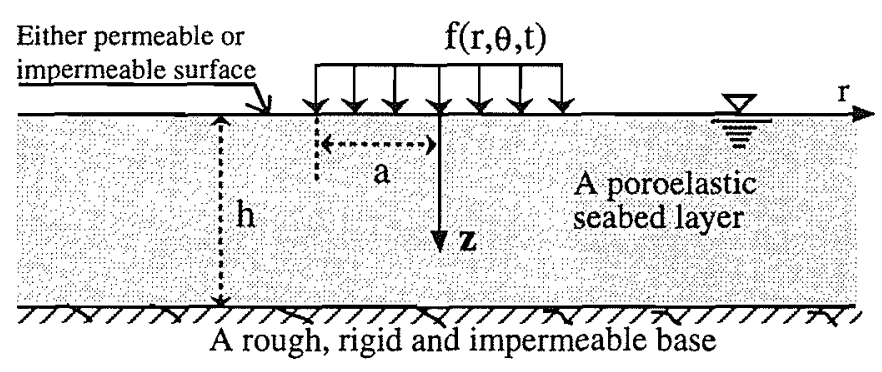

FrG. 1. A poroelastic seabed layer saturated with a compressible pore fluid loaded by a surface normal force.

gross physical properties of real saturated soil media. However, this theory is a physical and mathematical extension of the classical theory of elastostatics which accommodates the seepage flow described by Darcy's law. This theory provides a simplified description on dominant characteristics of the coupled interaction between the soil skeleton and the pore fluid of saturated soils (Mandel 1953; Cryer 1963). Furthermore, this theory represents a useful first approximation for the modeling of excess pore pressure in saturated seabed cohesive soils if the loads imparted to soil skeleton and (or) pore fluid lie within the working-load range and do not induce appreciable plastic deformation of soil skeleton and (or) turbulent flow of pore fluid.

Using Biot's theory of poroelasticity, this paper presents an analytical investigation on the quasi-static problem of the development of excess pore-fluid pressures in a poroelastic seabed layer and takes into account the influences of a compressible pore fluid. The seabed layer is modeled as a poroelastic medium of finite thickness saturated with a compressible pore fluid and resting on a rough rigid impermeable base. The study focuses on a parametric analysis of the development of excess pore-fluid pressures in the seabed layer whose surface has either free drainage or no drainage and is subjected to a normal surface traction (see Fig. 1). These two drainage conditions are the limiting cases that can offer the plausible "extreme estimates" for all the intermediate drainage conditions that can occur at the surface of the poroelastic seabed layer.

This class of consolidation problems related to a poroelastic medium of finite thickness has been examined by many investigators including Gibson et al. (1970), Booker (1974), Booker and Small (1982a, 1982b, 1987), Harnpattanapanich and Vardoulakis (1987), Sabin and Raman-Nair (1987), and Sabin (1989). In all these analytical investigations, however, it was assumed that the pore fluid is incompressible, which is a particular case of the generalized model of compressible pore fluids considered here. This assumption made it impossible to analytically examine the role of fluid compressibility on the consolidation response of excess pore pressure.

In recent years, purely computational schemes such as boundary element methods and finite element methods have been developed for the study of the consolidation responses of soils saturated with compressible fluids (Cheng and Liggett 1984; Cheng and Detournay 1988; and Chang and Duncan 1983). These numerical schemes, in particular the finite element methods, are powerful methods and, depending on the degree of sophistication, are capable of describing a variety of saturated soils. Various kinds of inelastic behaviour, nonlinearity, and anisotropy can be incorporated into a finite element analysis, provided that a realistic set of constitutive equations and material parameters can be determined via laboratory or in situ techniques. The reliability of these computational schemes can be greatly enhanced by the development of a group of benchmark analytical solutions. Such verified computational schemes can be applied with confidence to the examination of more complex problems involving practical geotechnical situations.Consequently, the objectives of this paper can be summarized as follows: (i) to extend the analytical modeling of excess pore pressure in a poroelastic seabed to include the compressibility of pore fluid; (ii) to present an alternative formulation for the development of analytical solutions governing the consolidation behaviour of a poroelastic layer; and (iii) to provide a group of benchmark results for the development and verification of existing and future numerical schemes.

\section{Governing equations}

In the ensuing, a brief account of the governing equations in Cartesian tensor notation is presented. The constitutive equations governing the quasi-static response of a poroelastic medium, which consists of an isotropic poroelastic soil skeleton and a compressible pore fluid, take the form

$$
\sigma_{i j}=\frac{2 \mu \nu}{1-2 \nu} \epsilon_{k k} \delta_{i j}+2 \mu \epsilon_{i j}-\frac{3\left(\nu_{\mathrm{u}}-\nu\right)}{B(1-2 \nu)\left(1+\nu_{\mathrm{u}}\right)} p \delta_{i j}
$$

$$
p=\frac{2 \mu B^{2}(1-2 \nu)\left(1+\nu_{\mathrm{u}}\right)^{2}}{9\left(\nu_{\mathrm{u}}-\nu\right)\left(1-2 \nu_{\mathrm{u}}\right)} \zeta_{\mathrm{v}}-\frac{2 \mu B\left(1+\nu_{\mathrm{u}}\right)}{3\left(1-2 \nu_{\mathrm{u}}\right)} \epsilon_{k k}
$$

where $\delta_{i j}$ is Kronecker's delta function, $p$ is the pore-fluid pressure, $\zeta_{\mathrm{V}}$ is the volumetric strain in the pore fluid, $\sigma_{i j}$ $(i, j=x, y, z)$ is the total stress tensor, $\epsilon_{\mathrm{kk}}$ is the volumetric strain of soil skeleton, and $\epsilon_{i j}$ are the soil skeleton strains defined by

$$
\text { [2] } \quad \epsilon_{i j}=\frac{1}{2}\left(u_{i, j}+u_{j, i}\right)
$$

and $u_{i}$ are the corresponding displacement components. The comma denotes a partial derivative with respect to a spatial variable. In the absence of body forces, the quasi-static equation of equilibrium takes the form

$$
\text { [3] } \sigma_{i j, j}=0
$$

The equation governing quasi-static fluid flow is defined by Darcy's law, which takes the form

$$
\text { [4] } v_{i}=-\kappa p_{. i}
$$

where $v_{i}$ is the specific discharge vector in the pore fluid, and $\kappa$ is a constant. The continuity equation associated with quasi-static fluid flow is

$$
\text { [5] } \frac{\partial \zeta_{\mathrm{v}}}{\partial t}+v_{i, i}=0
$$

where $t$ is time.

The above governing equations are characterized by five independent material parameters that can be represented by the drained and undrained Poisson's ratios $\nu$ and $\nu_{\mathrm{u}}$, respectively, shear modulus $\mu$, Skempton's pore-pressure coefficient $B$, and $\kappa\left(=k / \gamma_{w}\right.$, where $k$ is the coefficient of permeability and $\gamma_{w}$ is the unit weight of pore fluid) (Rice and Cleary 1976). In instances where the pore fluid is incompressible, the short-term "undrained" elastic response of the 
poroelastic medium is characterized by the constraints of $\nu_{u}=0.5$ and $B=1$. When the pore fluid is compressible, the undrained Poisson's ratio $v_{\mathrm{u}}$ and Skempton's pore-pressure coefficient $B$ have values whose limits are $-1<v<v_{\mathrm{u}}<0.5$ and $0<B<1$, respectively.

Based on the theory of Fourier integral transforms (Sneddon 1972), it can be shown that the following sets of solution representations exist for the field variables in a linear, isotropic, poroelastic layer of infinite lateral extent, saturated with a compressible pore fluid. Referred to either the temporal domain or the Laplace transform domain and in the cylindrical coordinate systems $(r, \theta, z)$ and $(\rho, \varphi, z)$, one has

[6]

$$
\begin{aligned}
& u(r, \theta, z, t)=\frac{1}{2 \pi} \int_{0}^{\infty} \int_{0}^{2 \pi} \frac{1}{\rho} \Pi w K \rho \mathrm{d} \varphi \mathrm{d} \rho, \\
& w(\rho, \varphi, z, t)=\frac{\rho}{2 \pi} \int_{0}^{\infty} \int_{0}^{2 \pi} \Pi * u K^{*} r \mathrm{~d} \theta \mathrm{d} r \\
& T_{z}(r, \theta, z, t)=\frac{1}{2 \pi} \int_{0}^{\infty} \int_{0}^{2 \pi} \Pi \tau K \rho \mathrm{d} \varphi \mathrm{d} \rho, \\
& \tau(\rho, \varphi, z, t)=\frac{1}{2 \pi} \int_{0}^{\infty} \int_{0}^{2 \pi} \Pi * T_{z} K^{*} r \mathrm{~d} \theta \mathrm{d} r
\end{aligned}
$$

$$
\begin{aligned}
& \boldsymbol{v}(r, \theta, z, t)=\frac{1}{2 \pi} \int_{0}^{\infty} \int_{0}^{2 \pi} \rho \Pi \boldsymbol{\Pi} K \rho \mathrm{d} \varphi \mathrm{d} \rho, \\
& \boldsymbol{\vartheta}(\rho, \varphi, z, t)=\frac{1}{2 \pi \rho} \int_{0}^{\infty} \int_{0}^{2 \pi} \Pi * v K * r \mathrm{~d} \theta \mathrm{d} r \\
& p(r, \theta, z, t)=\frac{1}{2 \pi} \int_{0}^{\infty} \int_{0}^{2 \pi} p_{\mathrm{w}} K \rho \mathrm{d} \varphi \mathrm{d} \rho, \\
& p_{\mathrm{w}}(\rho, \varphi, z, t)=\frac{1}{2 \pi} \int_{0}^{\infty} \int_{0}^{2 \pi} p K * r \mathrm{~d} \theta \mathrm{d} r
\end{aligned}
$$

where $0 \leq z \leq h, 0<t<\infty$, and the integrals are interpreted in the sense of a Cauchy principal value. The vectors in [6] are defined by $\boldsymbol{u}=\left(u_{r}, u_{0}, u_{z}\right)^{\mathrm{T}}, \mathbf{T}_{z}=\left(\sigma_{z, r}, \sigma_{z \theta}, \sigma_{z}\right)^{\mathrm{T}}$, $\boldsymbol{v}=\left(v_{r}, v_{0}, v_{2}\right)^{\mathrm{T}}, \boldsymbol{w}=\left(w_{1}, w_{2}, w_{3}\right)^{\mathrm{T}}, \boldsymbol{\tau}=\left(\tau_{1}, \tau_{2}, \tau_{3}\right)^{\mathrm{T}}$, and $\boldsymbol{\theta}=$ $\left(\vartheta_{1}, \vartheta_{2}, \vartheta_{3}\right)^{\mathrm{T}}$. The superscript $\mathrm{T}$ stands for the transpose of a matrix. $K^{*}$ and $\boldsymbol{\Pi}^{*}$ are, respectively, the complex conjugates of the Fourier matrix kernel functions $K$ and $\mathbf{I I}$ defined by

$$
\begin{aligned}
& \text { [7] } K=\mathrm{e}^{+i \rho r \sin (\theta+\varphi)}, \quad i=\sqrt{-1} \\
& \mathbf{I}=\left(\begin{array}{ccc}
+i \sin (\theta+\varphi) & +i \cos (\theta+\varphi) & 0 \\
+i \cos (\theta+\varphi) & +i \sin (\theta+\varphi) & 0 \\
0 & 0 & 1
\end{array}\right)
\end{aligned}
$$

By assuming the initial condition $\left.\zeta_{y}\right|_{t=0}=0$, the governing equations [1]-[5] can be rewritten as two sets of first-order ordinary differential equations in the Fourier and Laplace transform domains, i.e.,

$$
\text { [8] } \begin{aligned}
\frac{\mathrm{d}}{\mathrm{d} z} \mathbf{V}_{\mathrm{v}}(\rho, \varphi, z, s) & =\rho \mathbf{C}_{\mathrm{v}} \mathbf{V}_{\mathrm{v}}(\rho, \varphi, z, s) \\
\frac{\mathrm{d}}{\mathrm{d} z} \mathbf{V}_{\mathrm{u}}(\rho, \varphi, z, s) & =\rho \mathbf{C}_{\mathrm{u}} \mathbf{V}_{\mathrm{u}}(\rho, \varphi, z, s)
\end{aligned}
$$

where

$$
\mathbf{V}_{v}=\left(\begin{array}{c}
\hat{w}_{2} \\
\frac{1}{\mu} \hat{\tau}_{2}
\end{array}\right), \quad \mathbf{C}_{\mathrm{v}}=\left(\begin{array}{cc}
0 & 1 \\
1 & 0
\end{array}\right), \quad v_{k}=\frac{v_{\mathrm{u}}-\nu}{(1-v)\left(1-v_{\mathrm{u}}\right)}
$$$$
\text { [9] } \quad \mathbf{V}_{\mathrm{u}}=\left(\begin{array}{c}
\hat{w}_{1} \\
\hat{w}_{3} \\
\frac{1}{2 \mu} \hat{\tau}_{3} \\
\frac{1}{2 \mu} \hat{\tau}_{1} \\
\frac{1}{2 \mu \alpha_{\mathrm{d}}} \hat{p}_{\mathrm{w}} \\
\frac{1}{2 \mu \kappa \alpha_{d}} \hat{\vartheta}_{3}
\end{array}\right), \quad \alpha_{\mathrm{d}}=\frac{B\left(1+v_{\mathrm{u}}\right)}{3\left(1-v_{\mathrm{u}}\right)}
$$

$$
\begin{aligned}
& \mathbf{C}_{\mathrm{u}}=\left(\begin{array}{cccccr}
0 & -1 & 0 & 2 & 0 & 0 \\
\frac{v}{1-\nu} & 0 & \frac{1-2 \nu}{1-\nu} & 0 & v_{k} & 0 \\
0 & 0 & 0 & 1 & 0 & 0 \\
\frac{1}{1-\nu} & 0 & \frac{-v}{1-\nu} & 0 & \hat{v}_{k} & 0 \\
0 & 0 & 0 & 0 & 0 & -1 \\
\gamma^{2}-1 & 0 & 1-\gamma^{2} & 0 & -\gamma^{2} & 0
\end{array}\right) \\
& \gamma=\sqrt{\frac{1}{c} \frac{s}{\rho^{2}}+1,} \quad c=\frac{2 \mu B^{2}(1-\nu)\left(1+\nu_{u}\right)^{2} \kappa}{9\left(\nu_{u}-\nu\right)\left(1-\nu_{u}\right)}
\end{aligned}
$$

and the hat over a term denotes the Laplace transform with respect to $t$, and $s$ is the Laplace transform parameter.

By solving the ordinary differential equations, an algebraic solution representation of the field variables in the transform domain can be further obtained in terms of the field variables at the two boundary surfaces of a poroelastic layer, i.e.,

$$
\begin{aligned}
2 \mathbf{V}_{\mathrm{v}}(\rho, \varphi, z, s)= & {\left[\mathbf{A}_{\mathrm{q}} \mathrm{e}^{-\rho z}\right] \mathbf{V}_{\mathrm{v}}(\rho, \varphi, 0, s) } \\
& +\left[\mathbf{A}_{\mathrm{p}} \mathrm{e}^{\rho(z-h)}\right] \mathbf{V}_{\mathrm{v}}(\rho, \varphi, h, s) \\
2 \mathbf{V}_{\mathrm{u}}(\rho, \varphi, z, s)= & {\left[\mathbf{Q}_{\mathrm{q}} \mathrm{e}^{-\rho z}+\mathbf{Q}_{\mathrm{u}} \mathrm{e}^{-\rho \gamma z}\right.} \\
& \left.+\rho z \mathbf{Q}_{\mathrm{v}} \mathrm{e}^{-\rho z}\right] \mathbf{V}_{\mathrm{u}}(\rho, \varphi, 0, s) \\
& +\left[\mathbf{Q}_{\mathrm{p}} \mathrm{e}^{\rho(z-h)}+\mathbf{Q}_{\mathrm{s}} \mathrm{e}^{\rho \gamma(z-h)}\right. \\
& \left.+\rho(z-h) \mathbf{Q}_{\mathrm{u}} \mathrm{e}^{\rho(z-h)}\right] \mathbf{V}_{\mathrm{u}}(\rho, \varphi, h, s)
\end{aligned}
$$

where $0 \leq z \leq h$, the eight coefficient square matrices $\mathbf{A}_{\mathrm{p}}, \mathbf{A}_{\mathrm{q}}$, $Q_{p}$, etc., are nondimensional functions of $\gamma$ (see Appendix 1).

Substituting $z=0$ and $z=h$ in [10], one can further obtain a system of eight independent algebraic equations governing the relations among the 16 boundary variables at the two boundary surfaces of a poroelastic layer, i.e.,

$$
\begin{aligned}
& {\left[\mathbf{A}_{\mathrm{p}}-\mathbf{A}_{\mathrm{q}} \mathrm{e}^{-\rho / h}\right] \mathbf{V}_{\mathrm{v}}(\rho, \varphi, 0, s)} \\
& +\left[\mathbf{A}_{\mathrm{q}}-\mathbf{A}_{\mathrm{p}} \mathrm{e}^{-\rho h}\right] \mathbf{V}_{\mathrm{v}}(\rho, \varphi, h, s)=\mathbf{O} \\
& {[11] \quad\left[\mathbf{Q}_{\mathrm{p}}+\mathbf{Q}_{\mathrm{s}}-\mathbf{Q}_{\mathrm{q}} \mathrm{e}^{-\rho / h}-\mathbf{Q}_{\mathrm{t}} \mathrm{e}^{-\rho \gamma / h}\right.} \\
& \left.-\rho h \mathbf{Q}_{\mathrm{v}} \mathrm{e}^{-\rho / h}\right] \mathbf{V}_{\mathrm{u}}(\rho, \varphi, 0, s)+\left[\mathbf{Q}_{\mathrm{q}}+\mathbf{Q}_{\mathrm{t}}-\mathbf{Q}_{\mathrm{p}} \mathrm{e}^{-\rho / h}\right. \\
& \left.\quad-\mathbf{Q}_{\mathrm{t}} \mathrm{e}^{-\rho \gamma / h}+\rho h \mathbf{Q}_{\mathrm{u}} \mathrm{e}^{-\rho /}\right] \mathbf{V}_{\mathrm{u}}(\rho, \varphi, h, s)=\mathbf{O}
\end{aligned}
$$




\section{Consolidation problem}

The consolidation problem considered in this paper is related to the time-dependent response of a poroelastic seabed layer subjected to the action of a surface foundation load $f(r, \theta, t)$. The poroelastic seabed layer is saturated with a compressible pore fluid and is resting on a rough, rigid, and impermeable base (see Fig. 1). The surface of the poroelastic seabed is assumed to have either free drainage or no drainage. The initial reference state is assumed to be stress free, i.e., the analysis focuses on the excess stress states associated with the external loading. Consequently, the boundary conditions are as follows:

(1) At the upper surface $z=0$ of the consolidating seabed, one has the traction conditions

$$
\text { [12] } \begin{aligned}
\sigma_{z z}(r, \theta, 0, t) & =-f(r, \theta, t), \quad \sigma_{r z}(r, \theta, 0, t)=0 \\
\sigma_{\theta z}(r, \theta, 0, t) & =0
\end{aligned}
$$

and the drainage boundary conditions either

[13] $p(r, \theta, 0, t)=0 \quad$ for a completely pervious surface or

[14] $v_{z}(r, \theta, 0, t)=0 \quad$ for a completely impervious surface

(2) At the lower surface $z=h$ of the consolidating seabed, one has

[15] $\begin{array}{ll}u_{z}(r, \theta, h, t)=0, & u_{r}(r, \theta, h, t)=0, \\ u_{\theta}(r, \theta, h, t)=0, & v_{z}(r, \theta, h, t)=0\end{array}$

Consequently, using the [6]-[15], one can systematically express the variable $\hat{p}_{\mathrm{w}}(\rho, \varphi, z, s)$ in terms of the variable $\hat{\tau}_{3}(\rho, \varphi, 0, s)$, i.e.,

$$
\hat{p}_{\mathrm{w}}(\rho, \varphi, z, s)=\frac{B\left(1+\nu_{\mathrm{u}}\right)}{3\left(1-v_{\mathrm{u}}\right)} k_{0}(\gamma, \rho z, \rho h) \hat{\tau}_{3}(\rho, \varphi, 0, s)
$$

where $k_{0}(\gamma, \rho z, \rho h)$ can be algebraically obtained from [10] and [11], and

$$
\hat{\tau}_{3}(\rho, \varphi, 0, s)=-\frac{1}{2 \pi} \int_{0}^{\infty} \int_{0}^{2 \pi} \int_{0}^{\infty} f(r, \theta, t) \mathrm{e}^{-s t} K^{*} r \mathrm{~d} t \mathrm{~d} \theta \mathrm{d} r
$$

Substituting the solution of $\hat{p}_{w}(\rho, \varphi, z, s)$ into [6], one can express the excess pore pressure $p(\mathrm{r}, \theta, z, t)$ in the following. form:

$$
\begin{aligned}
& p(r, \theta, z, t)=\frac{B\left(1+\nu_{\mathrm{u}}\right)}{12\left(1-\nu_{\mathrm{u}}\right) \pi^{2} i} \\
& \int_{\omega-i \infty}^{\omega+i \infty} \int_{0}^{\infty} \int_{0}^{2 \pi} k_{0}(\gamma, \rho z, \rho h) \hat{\tau}_{3}(\rho, \varphi, 0, s) \mathrm{e}^{s t} K \rho \mathrm{d} \varphi \mathrm{d} \rho \mathrm{d} s
\end{aligned}
$$

where $\omega$ is a positive constant in the inverse Laplace transform. If the given variable $\hat{\tau}_{3}(\rho, \varphi, 0, s)$ has a Fourier series expansion of the form

$$
\hat{\tau}_{3}(\rho, \varphi, 0, s)=\sum_{m=-\infty}^{m=+\infty} \hat{\tau}_{3 m}(\rho, s) \mathrm{e}^{-i m \varphi}
$$

the excess pore pressure can also be further expressed in the form of a Fourier series, i.e.,

$$
\begin{aligned}
& p(r, \theta, z, t)=\frac{B\left(1+\nu_{u}\right)}{6\left(1-\nu_{u}\right) \pi i} \\
& \sum_{m=-\infty}^{m=+\infty} \int_{\omega-i \infty}^{\omega+i \infty} \int_{0}^{\infty} k_{0}(\gamma, \rho z, \rho h) \hat{\tau}_{3 m}(\rho, s) J_{m}(\rho r) \mathrm{e}^{s t} \rho \mathrm{d} \rho \mathrm{d} s \mathrm{e}^{i m \theta}
\end{aligned}
$$

where $J_{m}(\rho a)$ is the Bessel function of the $m$ th order.
For a constant normal load thàt is uniformly distributed over a circular area of radius $a$; one has

$$
f(r, \theta, t)=\frac{P_{z}}{\pi a^{2}} H(r-a) H(t)
$$

where $H()$ is a Heaviside step function. The variable $\hat{\tau}_{3}(\rho, \varphi, 0, s)$ can then be expressed in the following:

$$
\hat{\tau}_{3}(\rho, \varphi, 0, s)=\hat{\tau}_{30}(\rho, s)=-\frac{P_{z}}{\pi a} \frac{J_{1}(\rho a)}{\rho s}
$$

The excess pore pressure $p(\mathrm{r}, \theta, z, t)(=p(r, z, t))$ induced by the uniform and constant circular load is then expressed by

$$
\begin{aligned}
p(r, z, t)= & \frac{-P_{z}}{\pi a} \frac{B\left(1+\nu_{\mathrm{u}}\right)}{3\left(1-\nu_{\mathrm{u}}\right)} \frac{1}{2 \pi i} \\
& \int_{\omega \rightarrow \infty \infty}^{\omega+i \infty} \int_{0}^{\infty} k_{0}(\gamma, \rho z, \rho h) J_{0}(\rho r) J_{1}(\rho a) \frac{\mathrm{e}^{s t}}{s} \mathrm{~d} \rho \mathrm{d} s
\end{aligned}
$$

where $0^{+} \leq t<+\infty, 0 \leq z \leq h$, and $0 \leq r<+\infty$.

\section{Nondimensional expressions and limiting results}

By introducing the following nondimensional variables, i.e.,

$$
\begin{aligned}
& z_{1}=\frac{z}{a}, \quad r_{1}=\frac{r}{a}, \quad T=\frac{c t}{a^{2}}, \quad s_{1}=\frac{a^{2} s}{c} \\
& h_{1}=\frac{h}{a}, \quad \gamma_{1}=\sqrt{\frac{s_{1}}{\rho^{2}}+1}, \quad P_{0}\left(r_{1}, z_{1}, T\right)=\frac{\pi a^{2}}{B P_{z}} p\left(r_{1}, z_{1}, T\right)
\end{aligned}
$$

one can further express the excess pore pressure in the following nondimensional form, i.e.,

$$
\begin{aligned}
P_{0}\left(r_{1}, z_{1}, T\right) & =\frac{-\left(1+\nu_{\mathrm{u}}\right)}{3\left(1-\nu_{\mathrm{u}}\right)} \frac{1}{2 \pi i} \\
& \int_{\omega-i \infty}^{\omega+i \infty} \int_{0}^{\infty} k_{0}\left(\gamma_{1}, \rho z_{1}, \rho h_{1}\right) J_{0}\left(\rho r_{1}\right) J_{1}(\rho) \frac{\mathrm{e}^{s_{1} T}}{s_{1}} \mathrm{~d} \rho \mathrm{d} s_{1}
\end{aligned}
$$

where $0^{+} \leq T<+\infty, 0 \leq z_{1} \leq h_{1}$, and $0 \leq r_{1}<+\infty$.

It is instructive to first examine the limiting cases that pertain to the initial response as $t \rightarrow 0^{+}$and the final response as $t \rightarrow+\infty$ as well as the limiting response as $v \rightarrow v_{\mathrm{u}}$.

(1) For the poroelastic layer associated with a permeable surface, one has

[26] $\lim _{s \rightarrow+\infty} k_{0}(\gamma, \rho z, \rho h)= \begin{cases}0, & z=0 \\ k_{r}(\rho z, \rho h), & 0<z \leq h\end{cases}$

(2) For the poroelastic layer associated with an impermeable surface, one has

$$
\lim _{s \rightarrow+\infty} k_{0}(\gamma, \rho z, \rho h)=k_{r}(\rho z, \rho h)
$$
has

(3) For both permeable and impermeable surfaces, one

$$
\lim _{s \rightarrow+0} k_{0}(\gamma, \rho z, \rho h)=0, \quad \lim _{\nu \rightarrow v_{\mathrm{u}}} k_{0}(\gamma, \rho z, \rho h)=0
$$

where 


$$
k_{r}(x, y)=\frac{\mathrm{e}^{-x}\left\{(1+\alpha)\left(1+\mathrm{e}^{-2(2 y-x)}\right)+(1-\alpha)\left[(1-2 y) \mathrm{e}^{-2 y}+(1+2 y) \mathrm{e}^{-2(y-x)}\right]\right\}}{\left(\alpha^{2}-1\right)\left(1+\mathrm{e}^{-2 y}\right)^{2}-4 \mathrm{e}^{-2 y}\left[\alpha^{2}+(1-\alpha)^{2} y^{2}\right]}
$$

(1) For a fixed value of the undrained Poisson's ratio $v_{u}$,

By using above limiting results and the Tauberian theorems of Laplace transforms (Sneddon 1972), one can find that the excess pore pressure is zero either as $t \rightarrow+\infty$ or as $\nu \rightarrow \nu_{\mathrm{u}}$. The excess pore pressure has the following result as $t \rightarrow+0$, i.e.,

[30]

$$
\begin{aligned}
\lim _{t \rightarrow+0} P_{0}\left(r_{1}, z_{1}, T\right) & \\
& =-\frac{1+\nu_{\mathrm{u}}}{3\left(1-\nu_{\mathrm{u}}\right)} \int_{0}^{\infty} k_{r}\left(\rho z_{1}, \rho h_{1}\right) J_{0}\left(\rho r_{1}\right) J_{1}(\rho) \mathrm{d} \rho
\end{aligned}
$$

where $(i)$ for the permeable-surface case, $0<z_{1} \leq h_{1}$; and (ii) for the impermeable-surface case, $0 \leq z_{1} \leq h_{1}$.

The details of the numerical integration methodologies applicable to [25] and [30] were documented by Yue (1992). It was concluded from the numerical verification that the numerical integrations adopted in this study provide highly stable and accurate results in the time domain for the consolidation problems. In the following, attention will be focused on the presentation of a parametric study and numerical results of interest to offshore geotechnical engineering.

\section{Parametric study and numerical results}

In this section, parametric analyses are carried out to examine the time-dependent response of the excess pore pressure in a poroelastic seabed layer saturated with a compressible pore fluid. The time-dependent response of the excess pore pressure can be examined by considering separately the influences of the surface drainage conditions and the five material parameters $\left(\mu, \kappa, B, \nu, \nu_{\mathrm{u}}\right)$ as well as the length parameters $(a, h)$. The influence of the three material parameters $(\mu, \kappa, B)$ on the excess pore pressure can be analytically examined by using the nondimensional equations [25] and [30]. This is due to the fact that the nondimensional excess pore pressure $P_{0}\left(r_{1}, z_{1}, T\right)$ depends on only the drained and undrained Poisson's ratios $\left(\nu, \nu_{\mathrm{u}}\right)$ and the relative layer thickness $h_{1}$ as well as the surface drainage conditions. Owing to the limitation of space, the presentation will be restricted to the numerical results associated with the conditions where the relative layer thickness is unit, i.e., $h_{1}=1$, and the drained and undrained Poisson's ratios are the following, i.e.,

$$
\left(\nu, v_{u}\right)=(0,0.01),(0,0.2),(0,0.4),(0,0.5),(0.2,0.5),
$$

The selection of the seven sets of Poisson's ratios is based on the possible physical range of the values of Poisson's ratios $\left(0 \leq v<v_{u} \leq 0.5\right)$. The purpose of this selection is to obtain a compressive understanding of the time-dependent behaviour of the nondimensional excess pore pressure $P_{0}$ in the parametric study. The conventional time factor $T\left(=c t / a^{2}\right)$ is utilized in the presentation of the numerical results. It is noted that the conventional time factor $T$ is dependent on the Poisson's ratios $v$ and $v_{\mathrm{u}}$.

The time-dependent behaviour of the nondimensional excess pore pressure in the poroelastic seabed layer due to the constant circular load is plotted in Figs. $2-8$ by taking into account the effects of the two extreme surface drainage conditions. From these figures, we make the following observations, i.e., the variation in the nondimensional excess pore pressure with the conventional time factor $T$ is almost insensitive to the values of the drained Poisson's ratio (see Figs. 2-8).

(2) For a fixed value of the drained Poisson's ratio, the variation of the nondimensional excess pore pressure with the conventional time factor $T$ indicates the influence of the undrained Poisson's ratio. The lower the value of the undrained Poisson's ratio, the lower the magnitude of the excess pore pressure (see Figs. 2-8).

(3) The nondimensional excess pore pressures are almost equal to zero (less than 0.01) once the time factor $T$ reaches a certain value $T_{\mathrm{f}}$ which is independent on the two Poisson's ratios $\left(v, v_{\mathrm{u}}\right)$ and the locations $\left(r_{1}, z_{1}\right)$ in the poroelastic seabed layer. The values of $T_{\mathrm{f}}$ are estimated to be 2 and 30 for the completely permeable and the completely impermeable surface drainage conditions, respectively (see Figs. 2 and 3).

(4) Initial negative (tensile) excess pore pressures are induced in the exterior region of the cylindrical region beneath the constant circular load, i.e., $r_{1}>1$ and $0<z_{1}<1$. The magnitudes and regions of these negative excess pore pressure increase with the decrease of the undrained Poisson's ratio (see Figs. 6-8).

(5) For the completely permeable surface condition, the increase of excess pore pressure at the initial time stage, i.e., the Mandel-Cryer effect (Mandel 1953; Cryer 1963), occurs mainly in the interior region $\left(r_{1}<1\right)$ of the cylindrical region beneath the circular loaded area. For the completely impermeable surface condition, however, this effect occurs mainly in the exterior region $\left(r_{1}>1\right)$ of the circular cylindrical region (see Figs. 6-8). This additional increase of excess pore pressure at the initial stage decreases with the decrease in the difference of the two Poisson's ratios, i.e., $v_{\mathrm{u}}-v$.

(6) For the completely impermeable surface condition, the excess pore pressure is almost independent of the depth, i.e., the vertical coordinate $z_{1}$. This phenomenon is particularly evident for the conventional time factor $T>0.1$ (see Figs. 4 and 5).

(7) For a completely impermeable surface and for an undrained Poisson's ratio $\nu_{u}=0.5$, the nondimensional excess pore pressures at the initial time $T=0$ and at the surface loading centre $\left(r_{1}=0 ; z_{1}=0\right)$ are not equal to unity (see Figs. $4 e, 4 f, 5 e, 5 f$ ). In other words, the initial excess pore pressure at the impermeable surface is not equal to the applied normal pressure $P_{z} /\left(\pi a^{2}\right)$. This is due to the presence of a rigid rock base beneath the poroelastic seabed layer of finite thickness. If the poroelastic seabed layer is semi-infinite in extent, i.e., $h \rightarrow+\infty$, the initial excess pore pressure at the impermeable surface is equal to the applied normal pressure $P_{z} /\left(\pi a^{2}\right)$ (Yue and Selvadurai 1992). This result can be independently verified using the definition of excess pore pressure under undrained conditions, i.e., $p=\frac{B}{-3}\left(\sigma_{11}+\sigma_{22}+\sigma_{33}\right)$ and the data given by Poulos and Davis (1974) for a purely elastic layer of either finite thickness or semi-infinite extent.

(8) The drainage conditions at the surface of the poroelastic seabed layer have a significant effect on the 

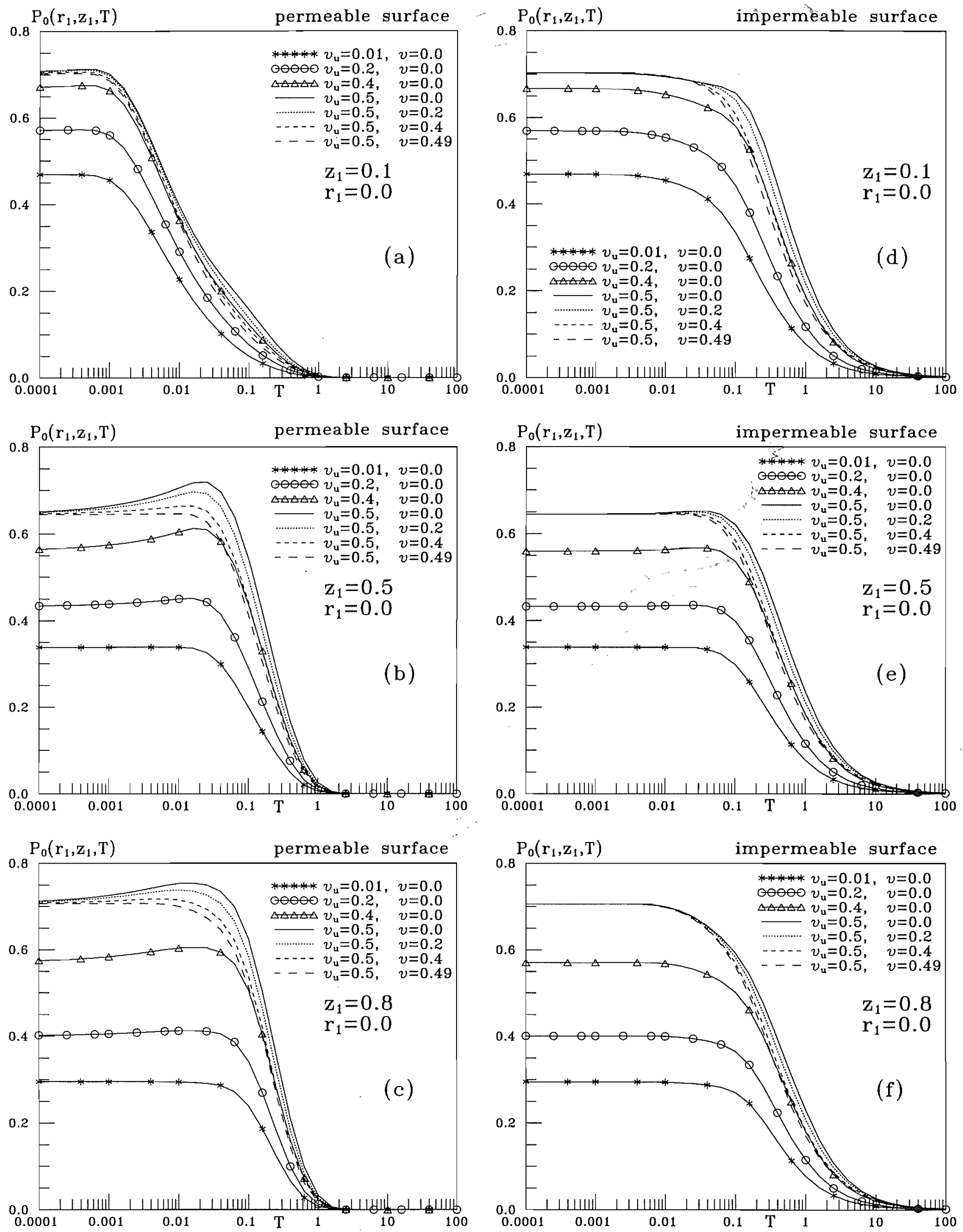

FIG. 2. Excess pore pressure in a poroelastic seabed layer vs. the nondimensional time factor $T$, where the nondimensional radial distance $r_{1}=0$. 

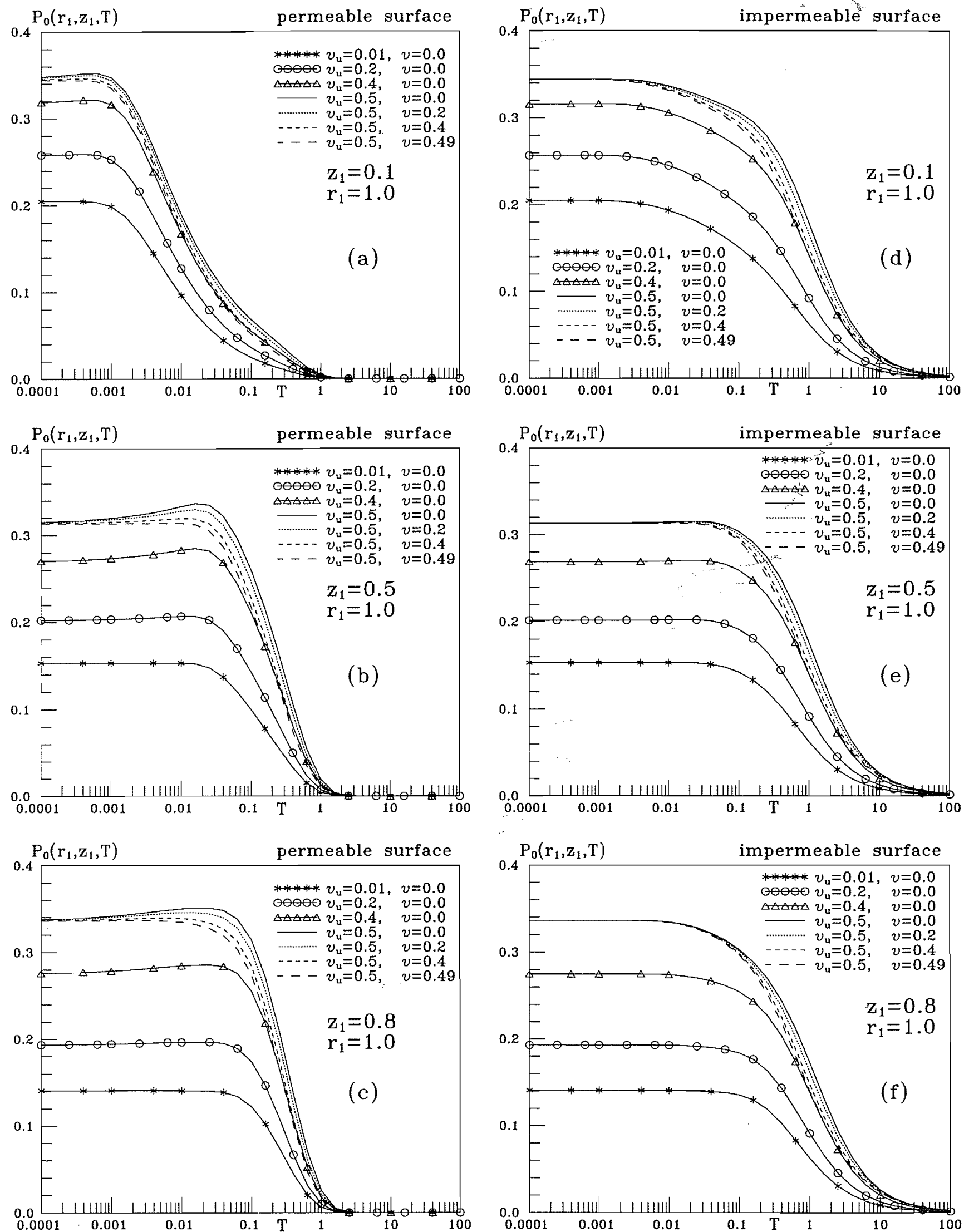

Fig. 3. Excess pore pressure in a poroelastic seabed layer vs. the non-dimensional time factor $T$, where the non-dimensional radial distance $r_{1}=1$. 

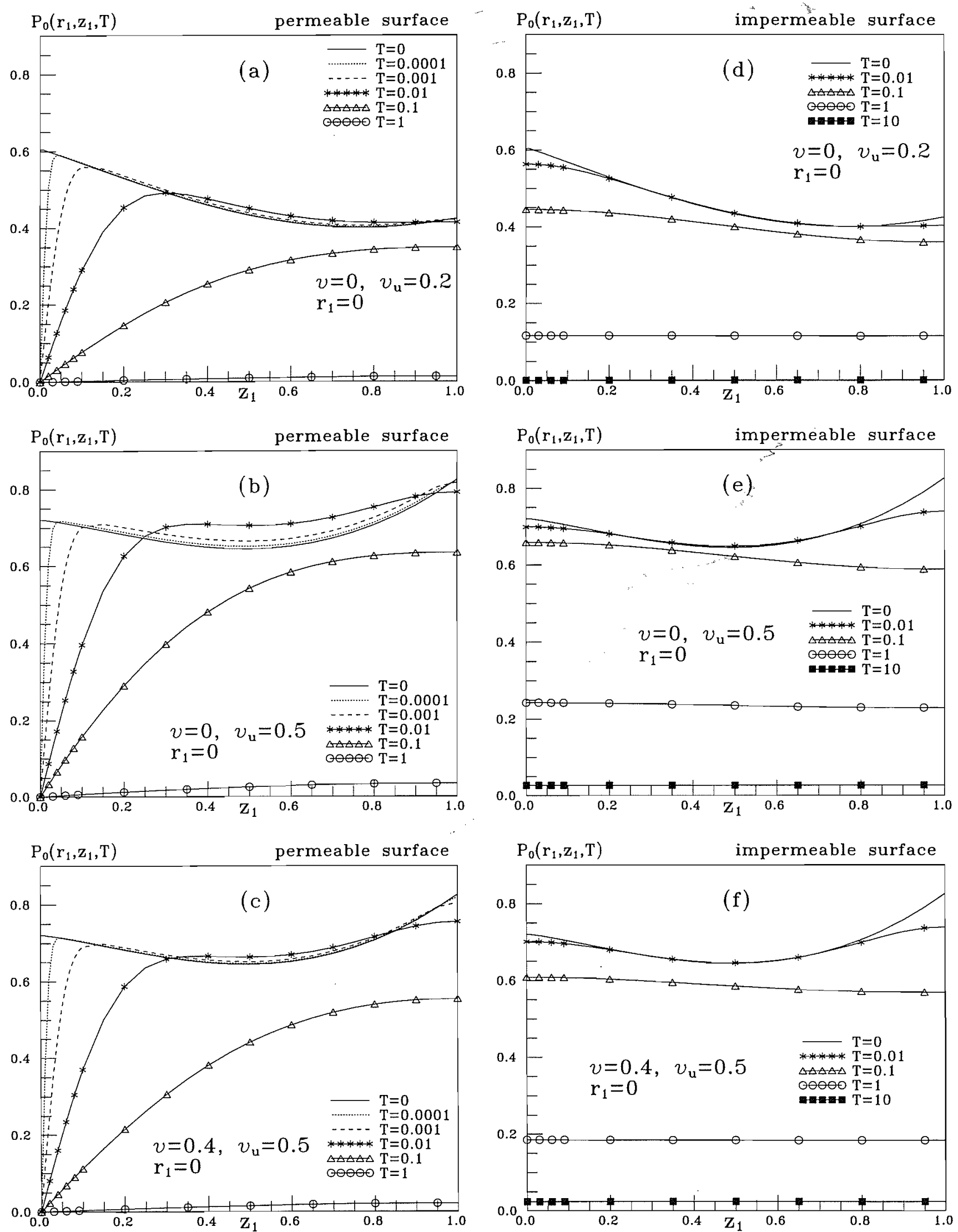

FIG. 4. Excess pore pressure in a poroelastic :eabed layer vs, the nondimensional depth $z_{1}$, where the nondimensional radial distance $r_{1}=0$. 

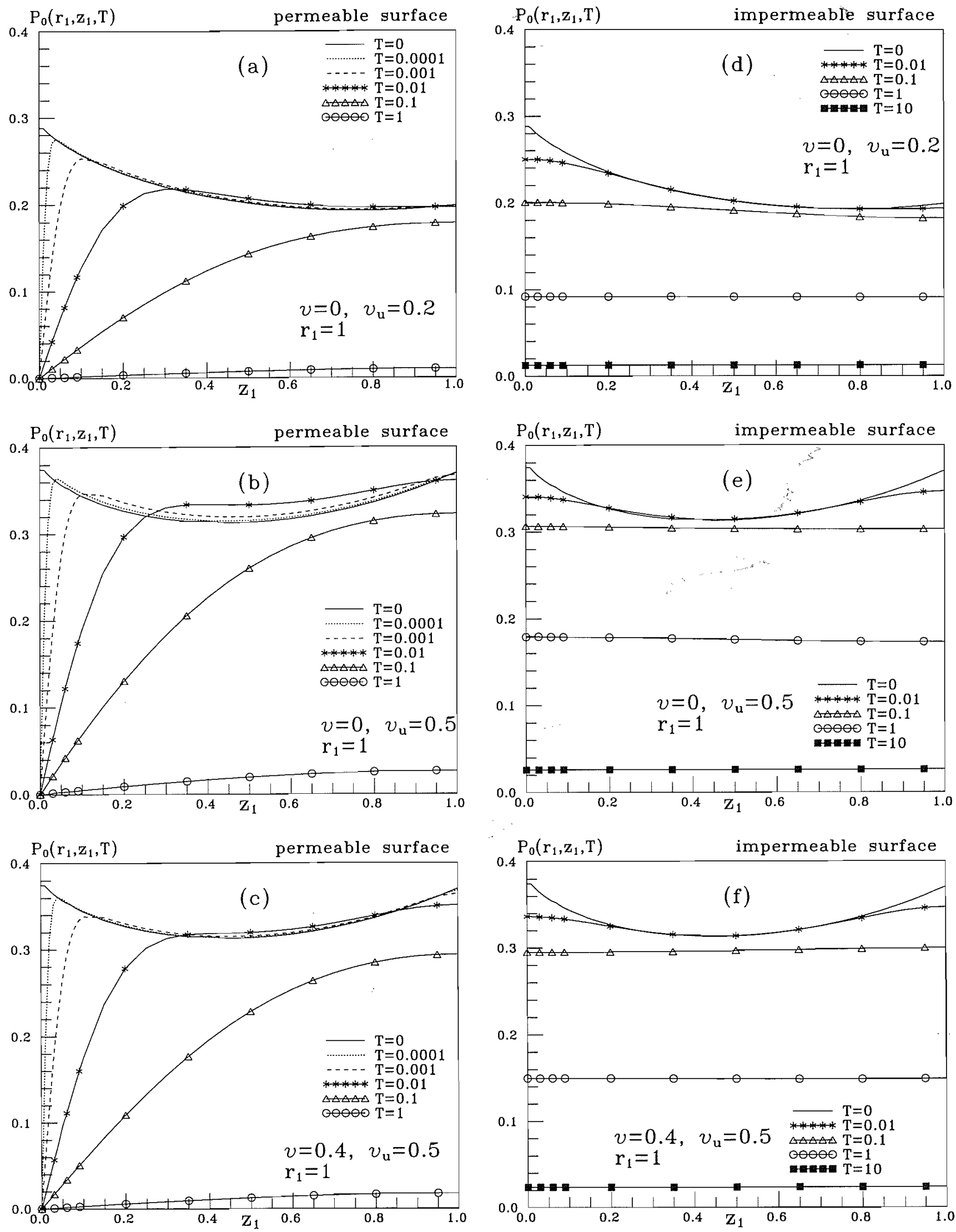

FIG. 5. Excess pore pressure in a poroelastic seabed layer vs. the nondimensional depth $z_{1}$, where the nondimensional radial distance $r_{1}=1$. 

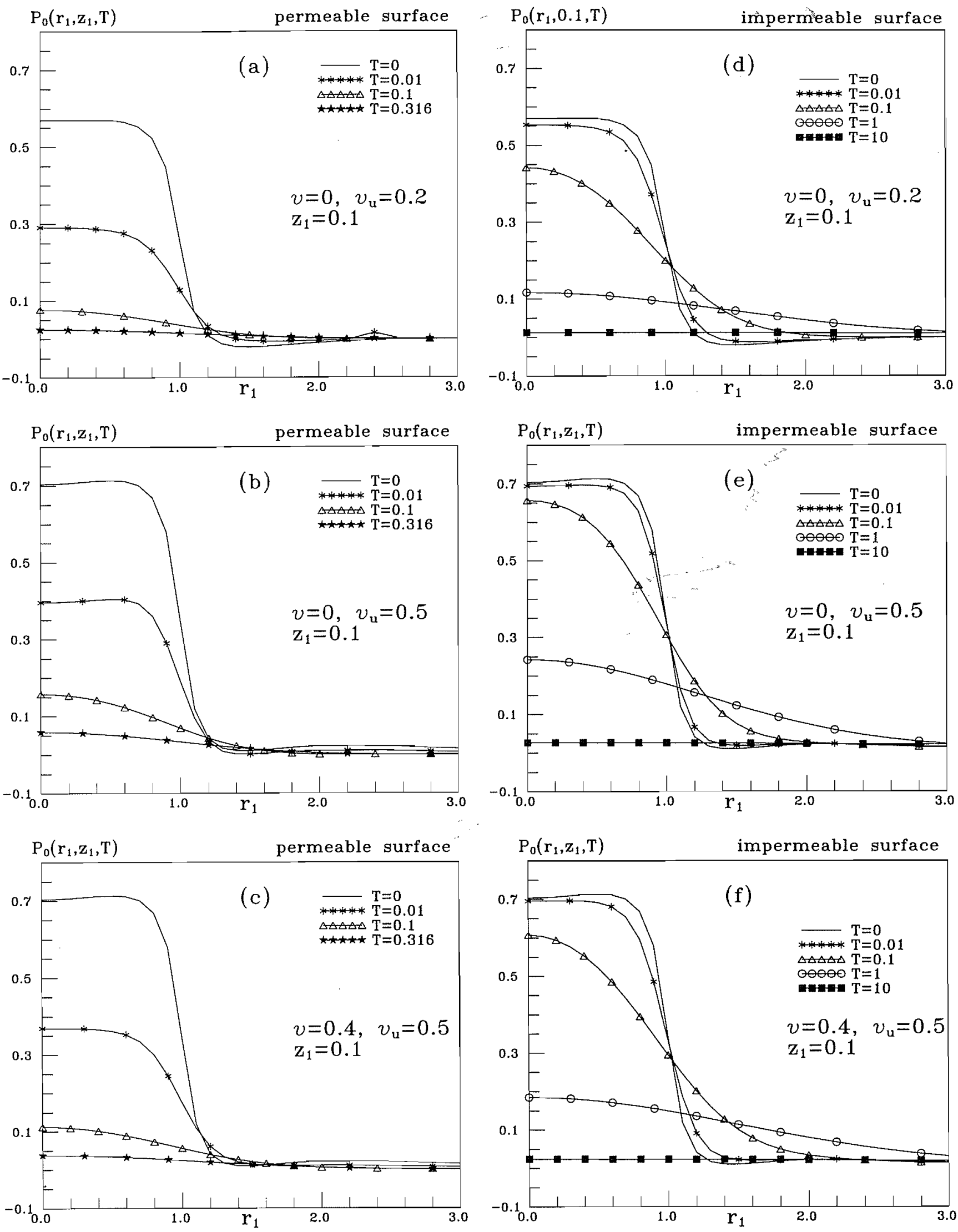

FIG. 6. Excess pore pressure in a poroelastic seabed layer vs. the nondimensional radial distance $r_{1}$, where the nondimensional depth $z_{1}=0.1$. 

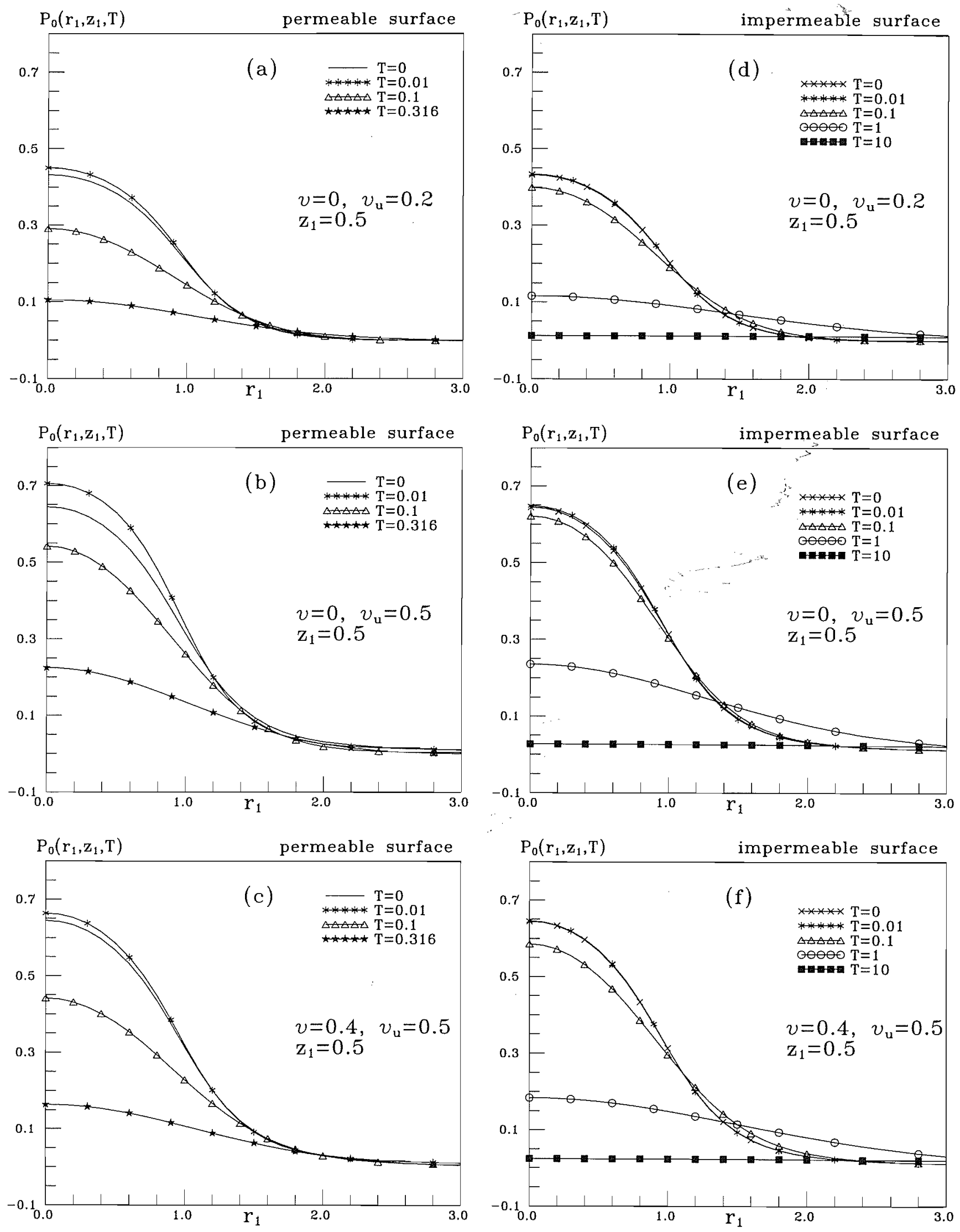

FIG. 7. Excess pore pressure in a poroelastic seabed layer vs. the nondimensional radial distance $r_{1}$, where the nondimensional depth $z_{1}=0.5$. 

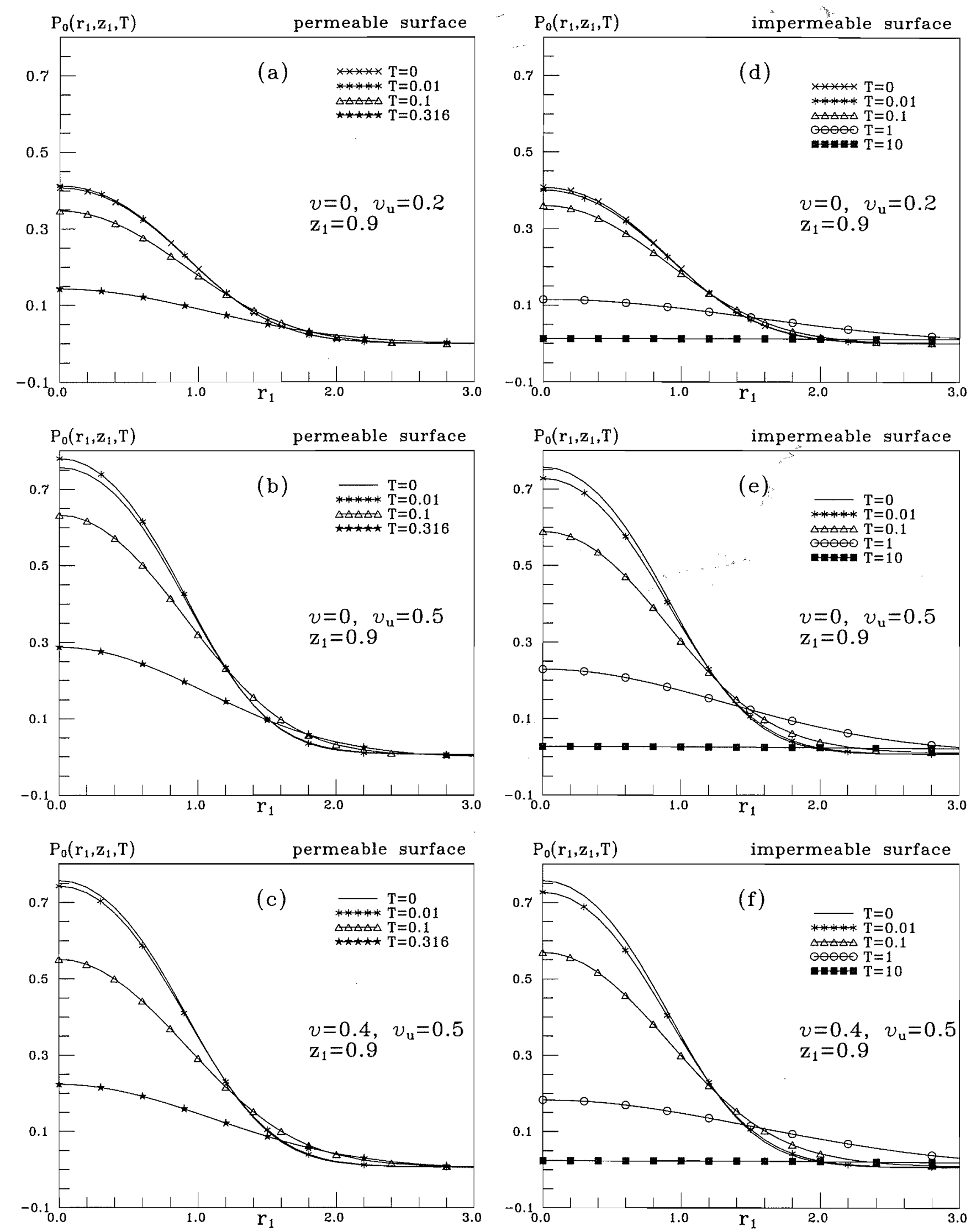

FIG. 8. Excess pore pressure in a poroelastic seabed layer vs. the nondimensional radial distance $r_{1}$, where the nondimensional depth $z_{1}=0.9$. 
dissipation rate of the excess pore pressure in the poroelastic seabed layer and, in particular, at the near-surface regions of the seabed layer (see Figs. 2-8).

(9) The variations of the nondimensional excess pore pressure $P_{0}$ versus the time factor $T$ are bounded by the results associated with the two sets of Poisson's ratios, i.e., $\left(v, v_{\mathrm{u}}\right)=$ $(0,0.5)$ and $(0,0.01)$ (Figs. 2 and 3$)$. For any other set of Poisson's ratios $\left(0 \leq v<\nu_{\mathrm{u}} \leq 0.5\right)$, the variations of $P_{0}$ versus $T$ can be estimated using the results presented in Figs. 2 and 3 .

Based on the above observations, the role of the five material parameters on the time-dependent behaviour of excess pore pressure in a poroelastic seabed layer can be summarized as follows.

(1) The magnitude of the excess pore pressure increases with the increase of undrained Poisson's ratio $\nu_{u}$ and increases proportionally with the increase in Skempton's pore-pressure coefficient $B$. The values of drained Poisson's ratio $v$ and shear modulus $\mu$ and $\kappa\left(=k / \gamma_{\mathrm{w}}\right)$ almost have no influence on the magnitude of the excess pore pressure.

(2) The dissipation rate of the excess pore pressure is determined by the combined effect of the five material parameters, i.e., the generalized coefficient of consolidation $c$. The total time $t_{\mathrm{f}}$ for the almost complete dissipation of the excess pore pressure can be estimated using the following equation:

$$
t_{\mathrm{f}}=\frac{a^{2}}{c} T_{\mathrm{f}}=\frac{9\left(\nu_{\mathrm{u}}-v\right)\left(1-v_{\mathrm{u}}\right) a^{2}}{2 \mu B^{2} \kappa(1-v)\left(1+\nu_{\mathrm{u}}\right)^{2}} T_{\mathrm{f}}
$$

where $T_{\mathrm{f}}$ is dependent on only the relative thickness $h_{1}$ of the poroelastic seabed layer and the drainage conditions at the surface of the poroelastic seabed layer. It can be observed from equation [32] that the total dissipation time $t_{\mathrm{f}}$ for the excess pore pressure is directly proportional to the difference between the two Poisson's ratios $\left(\nu_{u}-v\right)$ and inversely proportional to the values of $B^{2}$ and $\kappa$.

\section{Conclusions}

The time-dependent behaviour of excess pore pressure in a poroelastic seabed induced by a surface-normal load has been examined analytically by taking into account the effect of compressibility of the pore fluid. Based on the above results, it can be concluded that the generation of the excess pore pressure is reduced by the presence of a compressible pore fluid (i.e., smaller values of $v_{\mathrm{u}}$ and $B$ ) and that the dissipation rate of the excess pore pressure is determined by the generalized coefficient of consolidation, which is a combined effect of the five material parameters describing the characteristics of a poroelastic seabed layer. Furthermore, the group of numerical results presented in the paper can be used as benchmark solutions for the development and verification of purely computational schemes such as finite element and boundary element methods.

Biot, M.A. 1941. General theory of three-dimensional consolidation. Journal of Applied Physics, 12: 155-164.

Biot, M.A. 1956. General solution of the equations of elasticity and consolidation for a porous material. Journal of Applied Mechanics, 23: 91-95.

Booker, J.R. 1974. The consolidation of a finite layer subject to surface loading. International Journal of Solids and Structures, 10: 1053-1065.

Booker, J.R., and Small, J.C. 1982a. Finite layer analysis of consolidation I. International Journal for Numerical and Ana-: lytical Methods in Geomechanics, 6: 151-171.

Booker, J.R., and Small, J.C. 1982b. Finite layer analysis of consolidation II. International Journal for Numerical and Analytical Methods in Geomechanics, 6: 173-194.

Booker, J.R., and Small, J.C. 1987. A method of computing the consolidation behaviour of layered soils using direct numerical inversion of Laplace transforms. International Journal for Numerical and Analytical Methods in Geomechanics, 11: $363-380$.

Chang, C.S., and Duncan, J.M. 1983. Consolidation analysis for partly saturated clay by using an elastic-plastic effective stressstrain model. International Journal for Numerical and Analytical Methods in Geomechanics, 7: 39-55.

Cheng, A.H-D., and Detournay, E. 1988. A direct boundary element method for plane strain poroelasticity. International Journal for Numerical and Analytical Methods in Geomechanics, 12: $551-572$.

Cheng, A.H-D., and Liggett, J.A. 1984. Boundary integral equations methods for linear porous-elasticity with applications to soil consolidation. International Journal for Numerical Methods in Engineering, 20: 255-278.

Cryer, C.W. 1963. A comparison of the three-dimensional consolidation theories of Biot and Terzaghi. Quarterly Journal of Mechanics and Applied Mathematics, 16: 401-412.

Desai, C.S., and Saxena, S.K. 1977. Cônsolidation analysis of layered anisotropic foundations, International Journal for Numerical and Analytical Methods in Geomechanics, 1: 5-23.

Gibson, R.E., Schiffman, R.L., and Pu, S.L. 1970. Plane strain and axially symmetric consolidation of a clay layer on a smooth impervious base. Quarterly Journal of Mechanics and Applied Mathematics, 23: 505-520.

Harnpattanapanich, T., and Vardoulakis, I. 1987. Numerical Laplace-Fourier transform inversion technique for layeredsoil consolidation problems: II. Gibson soil layer. International Journal for Numerical and Analytical Methods in Geomechanics, 11: 103-112.

Mandel, J. 1953. Consolidation des sols (Étude mathématique). Géotechnique, 3: 287-299.

Poulos, H.G. 1988. Marine geotechnics. Unwin Hyman Ltd., London.

Poulos, H.G., and Davis, E.H. 1974. Elastic solutions for soil and rock mechanics. John Wiley \& Sons, Inc., New York.

Rendulic, L. 1936. Porenziffer and Porenwasserdrunk in Tonen. Der Bauingenieur, Berlin, 17(51/53): 559-564.

Rice, J.R., and Cleary, M.P. 1976. Some basic stress diffusion solutions for fluid-saturated elastic porous media with compressible constituents. Reviews of Geophysics and Space Physics, 14: 227-241.

Sabin, G.C.W. 1989. The behavior of a poroelastic seabed under normal and shear loads. Journal of Offshore Mechanics and Arctic Engineering, Transactions of the ASME, 111: 303-310.

Sabin, G.C.W., and Raman-Nair, W. 1987. The consolidation of a poroelastic seabed beneath an axisymmetric structure. In Proceedings of the 6th International Offshore Mechanics and Arctic Engineering Symposium, 1-6 March, Houston. Sponsored by the American Society of Mechanical Engineers. Vol. 1. pp. $403-410$.

Sneddon, I.N. 1972. The use of integral transforms. McGrawHill Company, New York.

Terzaghi, K. 1923. Die Berechnung der Durchlassigkeitzaiffer des Tones aus dem Verlaug der Hydrodynamischen Spannungsercheinungen. Akademie der Wissenchaften in Wein, Sitzungsberichte Mathematisch Naturwissenschaftlichen Klasse, Pt. IIa, 132(3/4): 125-138.

Yue, Z.Q. 1992. Mechanics of rigid disc inclusions in fluids saturated poroelastic media. Ph.D. thesis, Carleton University, Ottawa.

Yue, Z.Q., and Selvadurai, A.P.S. 1992. The role of Poisson's ratios on the consolidation response of soils. In Proceedings of 
the 46th Canadian Conference of Geotechnical Engineering, Toronto, 26-28 October.

Zaretskii, Yu. K. 1972. Theory of soil consolidation, [Translated from the Russián.] U.S. Department of Commerce, National Technical Information Service, Springfield,Va., TT70-50131.

\section{Appendix 1. The matrices in the fundamental solution representations}

$\mathbf{Q}_{\mathrm{s}}=\left(\begin{array}{cccccc}-\beta & \frac{1}{\gamma} \beta & \beta & \frac{-1}{\gamma} \beta & \beta & \frac{-1}{\gamma} \beta \\ -\gamma \beta & \beta & \gamma \beta & -\beta & \gamma \beta & -\beta \\ -\beta & \frac{1}{\gamma} \beta & \beta & \frac{-1}{\gamma} \beta & \beta & \frac{-1}{\gamma} \beta \\ -\gamma \beta & \beta & \gamma \beta & -\beta & \gamma \beta & -\beta \\ -1 & \frac{1}{\gamma} & 1 & \frac{-1}{\gamma} & 1 & \frac{-1}{\gamma} \\ \gamma & -1 & -\gamma & 1 & -\gamma & 1\end{array}\right), \quad \mathbf{Q}_{\mathrm{t}}=\left(\begin{array}{cccccc}-\beta & \frac{-1}{\gamma} \beta & \beta & \frac{1}{\gamma} \beta & \beta & \frac{1}{\gamma} \beta \\ \gamma \beta & \beta & -\gamma \beta & -\beta & -\gamma \beta & -\beta \\ -\beta & \frac{-1}{\gamma} \beta & \beta & \frac{1}{\gamma} \beta & \beta & \frac{1}{\gamma} \beta \\ \gamma \beta & \beta & -\gamma \beta & -\beta & -\gamma \beta & -\beta \\ -1 & \frac{-1}{\gamma} & 1 & \frac{1}{\gamma} & 1 & \frac{1}{\gamma} \\ -\gamma & -1 & \gamma & 1 & \gamma & 1\end{array}\right)$

$\mathbf{Q}_{p}=\left(\begin{array}{rrrrrr}1 & -\alpha & 0 & 1+\alpha & 0 & 0 \\ -\alpha & 1 & 1+\alpha & 0 & 0 & 0 \\ 0 & 1-\alpha & 1 & \alpha & 0 & 0 \\ 1-\alpha & 0 & \alpha & 1 & 0 & 0 \\ 1 & -1 & -1 & 1 & 0 & 0 \\ -1 & 1 & 1 & -1 & 0 & 0\end{array}\right)+\beta\left(\begin{array}{rrrrrr}1 & -1 & -1 & 1 & -1 & 1 \\ 1 & -1 & -1 & 1 & -1 & 1 \\ 1 & -1 & -1 & 1 & -1 & 1 \\ 1 & -1 & -1 & 1 & -1 & 1 \\ 0 & 0 & 0 & 0 & 0 & 0 \\ 0 & 0 & 0 & 0 & 0 & 0\end{array}\right)$

$\mathbf{Q}_{\mathrm{q}}=-\left(\begin{array}{cccccc}-1 & -\alpha & 0 & 1+\alpha & 0 & 0 \\ -\alpha & -1 & 1+\alpha & 0 & 0 & 0 \\ 0 & 1-\alpha & -1 & \alpha & 0 & 0 \\ 1-\alpha & 0 & \alpha & -1 & 0 & 0 \\ -1 & -1 & 1 & 1 & 0 & 0 \\ -1 & -1 & 1 & 1 & 0 & 0\end{array}\right)+\beta\left(\begin{array}{rrrrrr}1 & 1 & -1 & -1 & -1 & -1 \\ -1 & -1 & 1 & 1 & 1 & 1 \\ 1 & 1 & -1 & -1 & -1 & -1 \\ -1 & -1 & 1 & 1 & 1 & 1 \\ 0 & 0 & 0 & 0 & 0 & 0 \\ 0 & 0 & 0 & 0 & 0 & 0\end{array}\right)$

$\mathbf{Q}_{u}=(1-\alpha)\left(\begin{array}{rrrrrr}1 & -1 & -1 & 1 & 0 & 0 \\ 1 & -1 & -1 & 1 & 0 & 0 \\ 1 & -1 & -1 & 1 & 0 & 0 \\ 1 & -1 & -1 & 1 & 0 & 0 \\ 0 & 0 & 0 & 0 & 0 & 0 \\ 0 & 0 & 0 & 0 & 0 & 0\end{array}\right), \quad \mathbf{Q}_{v}=(1-\alpha)\left(\begin{array}{rrrrrr}-1 & -1 & 1 & 1 & 0 & 0 \\ 1 & 1 & -1 & -1 & 0 & 0 \\ -1 & -1 & 1 & 1 & 0 & 0 \\ 1 & 1 & -1 & -1 & 0 & 0 \\ 0 & 0 & 0 & 0 & 0 & 0 \\ 0 & 0 & 0 & 0 & 0 & 0\end{array}\right)$

$\mathbf{A}_{\mathrm{p}}=\left(\begin{array}{ll}1 & 1 \\ 1 & 1\end{array}\right), \quad \mathbf{A}_{q}=\left(\begin{array}{rr}1 & -1 \\ -1 & 1\end{array}\right), \quad \alpha=\frac{1-2 v_{u}}{2\left(1-\nu_{u}\right)}, \quad \beta=\frac{v_{u}-v}{(1-\nu)\left(1-v_{u}\right)\left(\gamma^{2}-1\right)}$

Appendix 2: List of symbols

$\sigma_{\mathrm{ij}} \quad$ the total stress tensor

$\epsilon_{\mathrm{ij}} \quad$ the strain tensor of soil skeleton

$\epsilon_{\mathrm{kk}}$ the volumetric strain of soil skeleton

$u_{i} \quad$ the displacement vector of soil skeleton

$p \quad$ the excess pore-fluid pressure

$\zeta_{v} \quad$ the volumetric strain in the pore fluid

$v_{i} \quad$ the specific discharge vector in the pore fluid

$w \quad$ the displacement vector $\boldsymbol{u}$ in the two-dimensional Fourier transform domain

$\tau$ the vertical stress vector $T_{z}$ in the two-dimensional Fourier transform domain

y the specific discharge vector $v$ in the two-dimensional Fourier transform domain

$p_{\mathrm{w}} \quad$ the excess pore-fluid pressure $p$ in the two-dimensional Fourier transform domain
$P_{0} \quad$ the nondimensional excess pore pressure

$f(r, \theta, t)$ the applied normal traction at the layer surface

$P_{z} \quad$ the total value of the applied normal traction over a circular area

$\sigma_{11} \quad$ the first principal stress at a point in an elastic layer

$\sigma_{22} \quad$ the second principal stress at a point in an elastic layer

$\sigma_{33}$ the third principal stress at a point in an elastic layer

$z \quad$ the depth from the top of the seabed layer

$x, y \quad$ the horizontal axes in a Cartesian coordinate system or substituting variables

$r, \theta$ the two horizontal axes in a cylindrical coordinate system

$\rho, \varphi \quad$ the two horizontal axes in a cylindrical coordinate 
system and in the two-dimensional Fourier transform domain

П, $K$

$T$

$T_{\mathrm{f}}$

$t_{\mathrm{f}}$

$\delta_{i j}$

$i$

$H()$

$J_{m}()$

the nondimensional radial distance $r / a$ from the loading centre

the nondimensional depth from the top of the seabed layer $z / a$

time

the Laplace transform parameter

Fourier matrix kernel functions

the nondimensional time factor $c t / a^{2}$

the time factor for almost zero excess pore pressure

the time for almost zero excess pore pressure

the Kronecker delta

the unit of an imaginary quantity $\sqrt{-1}$ or a subscript for the coordinates $x, y, z$

a Heaviside step function

the Bessel function of the order of $m(m=0, \pm 1$, $\pm 2, \ldots$ ) $\nu$

$\nu_{u}$

$\mu$

$B$

$\kappa$

$k$

$\gamma_{\mathrm{w}}$

$c$

$\alpha$

$\alpha_{d}$

$v_{k}$

$a$

$h$

$h_{1}$

$\omega$ the drained Poisson's' ratio

the undrained Poisson's ratio

shear modulus of soil

Skempton's pore-pressure coefficient

$k / \gamma_{w}$ (a constant)

the coefficient of permeability

the unit weight of pore fluid

the generalized coefficient of consolidation

$\left(1-2 v_{\mathrm{u}}\right) /\left[2\left(1-v_{\mathrm{u}}\right)\right]$

$B\left(1+\nu_{\mathrm{u}}\right) /\left[3\left(1-\nu_{\mathrm{u}}\right)\right]$

$\left(v_{\mathrm{u}}-v\right) /\left[(1-v)\left(1-v_{\mathrm{u}}\right)\right]$

the radius of the applied circular load

the thickness of the poroelastic seabed layer

the relative thickness of the poroelastic seabed layer $h / a$

a positive constant in the inverse Laplace transform

\title{
Fractal analysis of the slake durability test
}

\author{
Luis E. VALLEjo \\ Department of Civil Engineering, 949 Benedum Hall, University of Pittsburgh, Pittsburgh, PA 15261, U.S.A. \\ Received December 13, 1993 \\ Accepted July 12, 1994
}

Fragments of siltstone were subjected to the slake durability test. The profiles of the rock fragments experienced changes during the test as a result of saturation and abrasion-induced stresses. The profiles of the fragments were evaluated before and after the test using fractal analysis. Fractal analysis uses the concept of fractal dimension to calculate the roughness of profiles. Before the test, the profiles of the siltstone particles had an average fractal dimension equal to 1.1364. After the test, the average fractal dimension of the profiles became equal to 1.1187 . The change in fractal dimension was a reflection of the smoothing action on the particle profiles by the combined effects of abrasion and wetting and drying action that take place during the slake durability test.

Key words: durability, shape of rock fragments, fractal dimension.

Des fragments de siltstone ont été soumis à l'essai de durabilité par relâchement. Les profils des fragments de roche ont subi des changements au cours de l'essai résultant de la saturation et des contraintes induites par abrasion. Les profils des fragments ont été évalués avant et après l'essai au moyen de l'analyse fractale. L'analyse fractale utilise le concept de dimension fractale pour calculer la ruguosité des profils. Avant l'essai, les profils des particules de siltstone avaient un dimension fractale moyenne égale à 1,1364. Après l'essai, la dimension fractale moyenne des profils devenait égale à 1,1187 . Le changement de dimension fractale était le reflet de l'action d'aplanissement des profils de particule par les effets combinés d'abrasion, et de l'action de mouillage et séchage qui se produisent au cours de l'essai de durabilité par relâchement.

Mots clés : durabilité, forme des fragments rocheux, dimension fractale.

[Traduit par la rédaction]

Can. Geotech. J. 31, 1003-1008 (1994)

\section{Introduction}

Large masses of water-saturated rocks moving down a steep slope commonly generate a stream of broken debris which often moves at relatively high speeds over gentle slopes and for unexpectedly large distances (Hsu 1975). As the rock debris travels, abrasive forces cause changes in the profiles of the rock fragments. The profiles will be angular and rough at the beginning of the debris flow. However, as the debris flow moves down the slope, the profiles become rounded and smooth (Gerber and Scheidegger 1974). The Printed in Canada / Imprimé au Canada change in shape of the particle profiles will affect their frictional resistance and thus the distance that the rock debris can travel (Scheidegger 1973). Morris (1959) studied the influence of the shape (overall form), angularity, and surface texture of rock particles on the angle of frictional resistance mobilized by a mass of these particles when subjected to direct shear stress conditions. Morris discovered that the rougher the profiles of the rock particles, the higher the angle of internal friction between them (Table 1). Thus, the profile of rock particles had a marked influence on their 